\title{
Optical imaging of acute epileptic networks in mice
}

Edgar Guevara

Philippe Pouliot

Dang Khoa Nguyen

Frédéric Lesage 


\title{
Optical imaging of acute epileptic networks in mice
}

\author{
Edgar Guevara, ${ }^{a, b}$ Philippe Pouliot, ${ }^{\text {a,b }}$ Dang Khoa Nguyen, ${ }^{c}$ and Frédéric Lesage ${ }^{a, b}$ \\ á́cole Polytechnique de Montréal, Department of Electrical Engineering, C.P. 6079 succ. Centre-ville, Montréal, Quebec H3C 3A7, Canada \\ ${ }^{\text {b} M o n t r e a l ~ H e a r t ~ I n s t i t u t e, ~} 5000$ Bélanger Street, Montréal, Quebec H1T 1C8, Canada \\ 'University of Montreal Health Center (CHUM)-Hôpital Notre-Dame, Neurology Division, 1560 rue Sherbrooke est, Montreal, \\ Quebec H2L 4M1, Canada
}

\begin{abstract}
The potential of intrinsic optical imaging and resting-state analysis under anesthetized conditions as a tool to study brain networks associated with epileptic seizures is investigated. Using an acute model of epileptiform activity, the 4-aminopyridine model in live mice, we observe the changes in resting-state networks with the onset of seizure activity and in conditions of spiking activity. Resting-state networks identified before and after the onset of epileptiform activity show both decreased and increased homologous correlations, with a small dependence on seizure intensity. The observed changes are not uniform across the different hemodynamic measures, suggesting a potential decoupling between blood flow and metabolism in the low-frequency networks. This study supports the need for a more extensive investigation of epileptic networks including more than one independent hemodynamic measurement. ( ) The Authors. Published by SPIE under a Creative Commons Attribution 3.0 Unported License. Distribution or reproduction of this work in whole or in part requires full attribution of the original publication, including its DOI. [DOI: 10.1117/1 .JBO.18.7.076021]
\end{abstract}

Keywords: functional connectivity; resting state; biomedical imaging; optical intrinsic signals; laser speckle contrast; 4-aminopyridine; animal models of epilepsy.

Paper 130226R received Apr. 9, 2013; revised manuscript received Jun. 19, 2013; accepted for publication Jun. 27, 2013; published online Jul. 25, 2013.

\section{Introduction}

Epilepsy is a group of neurological disorders where the abnormal synchrony of discharge in large ensembles of neurons results in an epileptic seizure (ictus), which over time may have serious consequences. ${ }^{1}$ Epilepsy is one of the most common major neurological disorders, affecting $0.4 \%$ to $0.8 \%$ of the population. ${ }^{2}$ The current method to visualize abnormal epileptiform discharges in patients with epilepsy is to perform an electroencephalogram (EEG), a technique that uses electrodes to record the spontaneous electrical activity of the brain. ${ }^{3}$ Epileptiform abnormalities are usually divided into (a) "interictal" discharges that are brief (milliseconds) asymptomatic paroxysmal EEG transients clearly distinguished from background; and (b) "ictal" discharges that are sudden focal rhythmic activity with characteristic patterns of evolution (with respect to amplitude, frequency, and spatial distribution) lasting at least several seconds. These ictal discharges are generally associated with clinical seizure manifestations.

Functional imaging based on cerebral perfusion such as combined functional MRI-EEG (fMRI-EEG) ${ }^{4}$ and functional near-infrared spectroscopy (fNIRS-EEG) ${ }^{5}$ have recently been explored for their potential at identifying the epileptic focus as well as study the coupling between brain regions involved in the epileptic network. While hemodynamic changes triggered by epileptic events have potential for localization, neuroimaging observations in patients remain confounded in several ways. An overarching reason is that the hemodynamic response is often not restricted to the epileptogenic focus but spreads across a distributed network of brain structures, with complicated dynamics. ${ }^{6,7}$ For example, the presence of mirror activation ${ }^{8}$

Address all correspondence to: Frédéric Lesage, Dept. Génie Électrique, École Polytechnique de Montréal, C.P. 6079 succ. Centre-ville, Montréal, Quebec H3C 3A7, Canada. Tel:+1-514-340-4711x7542; Fax: +1-514-340-4611; E-mail: frederic.lesage@polymtl.ca sometimes makes lateralization difficult. Even when there is a clear localized focus, the temporal aspects between oxyhemoglobin $\left(\mathrm{HbO}_{2}\right)$, deoxyhemoglobin ( $\left.\mathrm{HbR}\right)$, cerebral blood flow $(\mathrm{CBF})$, volume, and oxygen metabolism are still very intricate. Interictal discharges, and seizures even more so, are large neuronal discharges requiring an increased metabolic consumption ${ }^{9}$ and thus accompanied by a large increase of perfusion, rendering the interpretation of fMRI and fNIRS data difficult, given the complicated relationship between the various quantities of interest.

The term functional connectivity is applied to a variety of studies that examine coherent inter-region+al correlations of neuronal activity during both cognitive tasks and rest. ${ }^{10}$ It is a technique sensitive to spatial and temporal synchronicity of fluctuations in neural activity. ${ }^{11}$ This technique has been widely used with EEG measures in several studies that found changes in synchronicity before and after the seizure. ${ }^{12-16}$ Interictal determination of EEG functional connectivity (fcEEG) has been reported in patients with mesial temporal lobe epilepsy, ${ }^{17}$ as well as in epileptic children. ${ }^{18}$ Furthermore, fcEEG has been shown to be a predictor of epilepsy diagnosis. ${ }^{19}$ The application of functional connectivity using hemodynamic measurements to epilepsy is motivated both by observations of a distributed network of activations during epileptic events and by recent studies with resting-state functional magnetic resonance imaging, ${ }^{11,20-22}$ which have documented an altered functional connectivity in several functional networks in epileptic patients. In the first of these studies, investigations in patients with left temporal lobe epilepsy have reported a significant decrease of connectivity in language-related areas during resting state when compared to controls. ${ }^{11}$ Separate studies of epileptic networks documented a decrease of functional connectivity in the self-referential, somatosensory, visual, and auditory networks, ${ }^{22}$ as well as in the default mode network ${ }^{20,23,24}$ and in the dorsal attention network. ${ }^{25}$ The distributed nature of activation during epileptic 
seizures, the heterogeneity within the patient population and nonlinear coupling ${ }^{26,27}$ between vascular components during large neuronal discharges support investigating these phenomena in controlled animal models to better characterize network changes with epileptic events.

We adapted in this work a recently developed technique that combines functional connectivity mapping with optical intrinsic signal (fcOIS) imaging. ${ }^{28,29}$ This allowed measuring the magnitude of bilateral functional connectivity after the induction of focal seizures in the somatosensory cortex, using a seed regionbased cross-correlation analysis. Since all procedures were carried out under anesthetized condition, the resting anesthetized state is simply referred as resting state, for the sake of simplicity and clarity. In addition to the $\mathrm{HbO}_{2}$ contrast previously used in Refs. 28 and 29, we examined HbR and also blood flow measured simultaneously with laser speckle imaging to provide an extended set of measures characterizing hemodynamic changes during large neuronal discharges. This multimodal approach with optical and electrical recordings provides a comprehensive depiction of the resting-state activity, before and after the injection of the neurotoxin. Optical imaging based on intrinsic signals (OIS) and laser speckle blood flow imaging map hemodynamic variables all over the convexity of the cortex, and electrophysiology recordings give a precise measurement of local field potential (LFP) in the area surrounding the epileptic focus. Injection of the neurotoxin 4-aminopyridine (4-AP) in the left somatosensory cortex was used to produce seizure-like epileptiform discharges (later simply referred to as seizures) resulting from neuronal hyperactivity in cortical neurons. ${ }^{30-32}$ This model offers the possibility to make a comparison of the healthy cortex and the epileptic cortex on the same experimental animal. Motivations for the current study are two-fold: (1) understanding the modulation of brain networks at an early stage in ictogenesis is important to gain a better understanding of the pathophysiology of the disease $\mathrm{e}^{20,21,33}$ and may help uncover the mechanisms by which these brain networks are altered. (2) A long-term motivation is to evaluate the usefulness of resting-state analyses to support epileptic focus localization or seizure detection. Our aim in this work was to provide a proof-of-principle study using intrinsic optical imaging on acute epileptiform events, with a perspective of translating these techniques to a more chronic animal model of epilepsy and in conditions where anesthesia is not required.

\section{Methods}

\subsection{Animal Preparation}

All surgical procedures, performed according to the recommendations of the Canadian Council on Animal Care, were approved by the Animal Research Ethics Committee of the Montreal Heart Institute. Nine male C57BL/6 mice $(8.9 \pm 0.2$ weeks old, $23.3 \pm 2.3 \mathrm{~g}$ weight, Charles River, Wilmington, MA) were anesthetized via intraperitoneal injections of urethane $(2 \mathrm{mg} / \mathrm{g}$ body weight) in a $10 \%$ (wt/vol) saline solution. Body temperature was maintained at $37^{\circ} \mathrm{C}$ with a feedback controlled heating blanket (MouseSTAT, Kent Scientific, Torrington, CT).

A tracheotomy was done in order to avoid respiratory distress. ${ }^{34}$ Scalp was carefully removed and a stereotaxically guided burr-hole was made over the left somatosensory cortex [co-ordinates $1 \mathrm{~mm}$ lateral to midline, $1 \mathrm{~mm}$ caudal to bregma, Fig. 1(b)] for the injection of 4-AP and electrophysiological monitoring, using a 22-gauge needle.
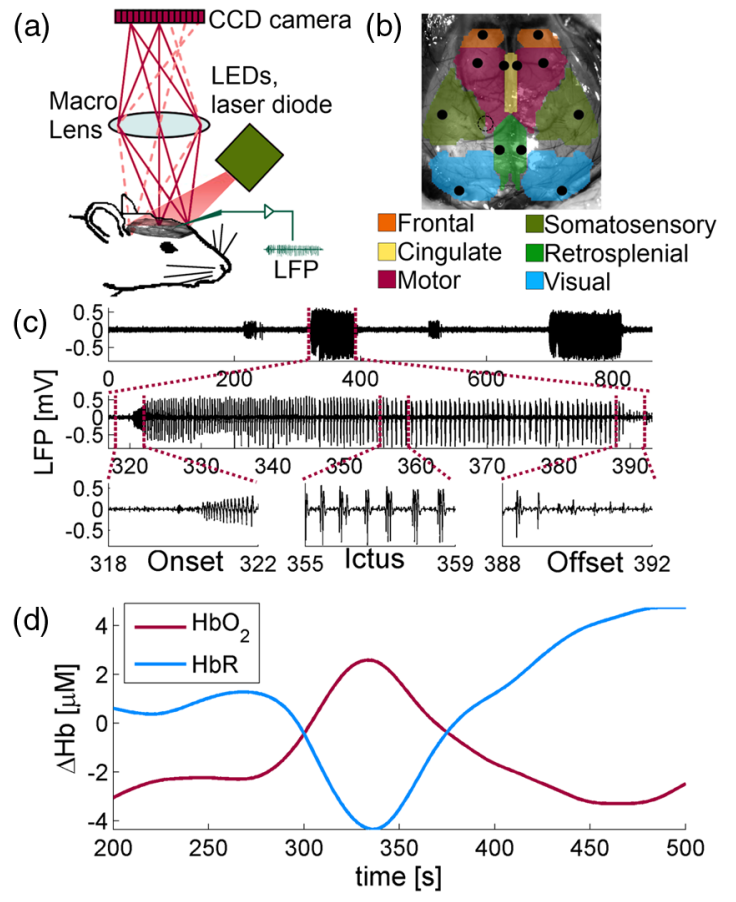

Fig. 1 (a) Overview of the intrinsic signal optical imaging system. LEDs and laser diode are time-multiplexed and synchronized to the acquisition system. A tungsten electrode is used to record local field potential (LFP) on the left somatosensory cortex. (b) Functional regions on the mouse cortex and seed placement and size, manually constructed from the work of Bero et al. ${ }^{28}$ Dotted circle shows the placement of the LFP electrode. (c) Electrophysiology of 4-AP induced seizure. Top: example showing some ictal discharges after an injection. 4-AP injection was finished at time 0. Middle: zoom on a single ictal discharge. Bottom: expanded view showing the onset of the discharge, the intermediate phase and the offset. (d) Filtered time traces of oxyhemoglobin $\left(\mathrm{HbO}_{2}\right)$ and deoxyhemoglobin $(\mathrm{HbR})$ at the epileptic focus [dotted circle in (b)], during the seizure.

Mice were placed on a stereotaxic apparatus and subcutaneous electrocardiogram was recorded with a single-lead electrocardiography (ECG) amplifier (amp-b01, emka Technologies, Paris). The amplified $(1000 \times$ gain $)$ and filtered signal $(0.2$ to $500 \mathrm{~Hz}$ ) was sampled at $1 \mathrm{kHz}$. All vital signals were digitized by a data acquisition card (NI-USB 6353, National Instruments, Austin, TX) controlled by a custom-made graphical user interface developed in LabView (National Instruments), which also controlled the image acquisition. To prevent drying of the exposed skull, a custom chamber made of bone wax was adhered to the skull with ultrasound gel and filled with mineral oil, after positioning the microelectrode.

\subsection{Epilepsy Model and Electrophysiology}

Epileptiform activity was achieved according to the procedures described by Zhao et al., ${ }^{31,32}$ briefly described below. Focal seizures were induced by the injection of $500 \mathrm{~nL}$ of the $\mathrm{K}^{+}$-channel blocking agent 4-AP (A78403, Sigma-Aldrich, St. Louis, MO) solution at $15 \mathrm{mM}$, through a glass micropipette with a microsyringe pump controller (uMC4, World Precision Instruments, Sarasota, FL). The volume was injected at a rate of $50 \mathrm{~nL} / \mathrm{min}$. The borosilicate glass pipette $(1 \mathrm{~mm}$ outer diameter, $0.7 \mathrm{~mm}$ inner diameter, $75 \mathrm{~mm}$ long, World Precision Instruments, New Haven, CT) was horizontally pulled on a programmable pipette puller (P-2000, Sutter Instruments, Novato, CA). 
Following injection, the glass pipette was removed and then a tungsten microelectrode $(0.5$ to $2 \mathrm{M} \Omega$ ) was placed at a depth of $\sim 500 \mu \mathrm{m}$ into the cortex, in order to record extracellular LFP at the injection site. The signal was filtered between 10 and $5000 \mathrm{~Hz}$, amplified 1000 times with a microelectrode AC amplifier (model 1800, A-M systems, Sequim, WA), and then digitized at $10 \mathrm{kHz}$. LFP data was further filtered between 0.2 and $130 \mathrm{~Hz}$ using an order 4 Butterworth digital filter in postprocessing. An example of typical epileptiform activity is shown in Fig. 1(c).

\subsection{Optical Recording System}

OIS were acquired with a 12-bit charge-coupled device (CCD) camera (Pantera 1M60, DS-21-01M60-12E, Teledyne Dalsa, Waterloo, ON) with a physical pixel size on chip of $12 \mu \mathrm{m}$ and full resolution of $1024 \times 1024$ pixels. The setup used in this work is depicted in Fig. 1(a).

A custom-made interface controls the camera and records images and vital signs, while synchronizing acquisition and illumination. A macro lens (105 mm f/2.8 max, Sigma Corp., Ronkonkoma, NY) with small focal depth $(350 \mu \mathrm{m})$ was used. Reflectance images of the brain cortex were recorded with timemultiplexed illumination $(525,590,625 \mathrm{~nm})$ produced by $10 \mathrm{~W}$ LEDs (LZ4-00MA00, Led Engin, San Diego, CA). Illumination for speckle imaging was provided by $90 \mathrm{~mW}, 785 \mathrm{~nm}$ laser diode (L785P090, Thorlabs, Newton, NJ) and aperture was adjusted to $\mathrm{f} / 8$, so that the pixel size and speckle size were matched. The four temporally multiplexed wavelengths led to a full-frame rate of $5 \mathrm{~Hz}$. A $2 \times 2$ binning on camera was performed to allow continuous data streaming to hard drive. Illumination was further adjusted so that no part of the brain was under- or oversaturated by any of the wavelengths. The exposure time of the camera was set to $10 \mathrm{~ms}$. The setup was mounted on an optic table with tuned damping (RS 2000, Newport, Irvine, CA) to avoid spurious signals from vibrations.

\subsection{Optical Imaging of Intrinsic Signals}

The analysis of spectroscopic images was based on previously published work. ${ }^{35,36}$ In short, reflectance images from each LED wavelength were recorded with the CCD camera and interpreted as changes in attenuation (optical density) $\Delta \mathrm{OD}=\log \left(I_{0} / I\right)$, where $I$ is the reflected light intensity and $I_{0}$ is the incident light intensity. Relative changes in $\mathrm{HbO}_{2}$ and $\mathrm{HbR}$ were found using the modified Beer-Lambert law ${ }^{37}$ and a Moore-Penrose pseudoinverse:

$$
\Delta \mathrm{OD}(\lambda, t)=\sum_{i} \epsilon_{i}(\lambda) C_{i}(t) D(\lambda)
$$

The differential path length factor, $D(\lambda)$, was taken from Ref. 38 and values out of the 560- to 610-nm range were extrapolated from Ref. 39. Total hemoglobin baseline concentration of $100 \mu \mathrm{M}$ with $60 \%$ oxygen saturation was assumed ${ }^{38}$ for the spectroscopic analysis. The hemoglobin extinction coefficients were obtained from Ref. 40, and the reflectance values were corrected for the spectral response of the CCD camera and convolved with the LEDs' spectral power distribution. ${ }^{41}$

Images of each chromophore $\left(\mathrm{HbO}_{2}, \mathrm{HbR}\right)$ were spatially smoothed with a Gaussian kernel of $11 \times 11$ pixels $(0.6 \times$ $0.6 \mathrm{~mm})$ with a 5-pixel standard deviation $(0.3 \mathrm{~mm})$. An anatomical image was recorded with illumination at $525 \mathrm{~nm}$, and then the region corresponding to brain was manually selected using a closed spline curve to create a brain mask. All further processing was performed only on those pixels belonging to the brain mask.

\subsection{Speckle Contrast Imaging}

In addition to imaging changes in hemoglobin concentrations, changes in $\mathrm{CBF}$ were computed by laser speckle contrast imaging. Speckle originates from the random interference of multiple backscattered coherent light. In the presence of moving scatterers the interference field fluctuates, creating intensity variations, known as speckle patterns. ${ }^{38,42,43}$ When integrated over the exposure time of the camera, the speckle pattern becomes blurred in areas of increased blood flow. Images of blood flow were obtained by measuring the spatial contrast of the speckle $C$, defined as the ratio of the standard deviation to the average intensity $\sigma /\langle I\rangle$. The contrast is a function of the CCD camera exposure time $T$ and it is related to the correlation time $\tau_{C}$ of the speckle, which is assumed to be inversely proportional to the speed of the scattering particles. ${ }^{44}$ A window of $5 \times 5$ pixels $(0.3 \times 0.3 \mathrm{~mm})$ was used to compute the speckle contrast images and the relative changes in blood flow were obtained with the following formula:

$$
C=\frac{\sigma}{\langle I\rangle}, \quad \frac{2 \Delta C}{C_{0}} \approx \frac{\Delta V}{V_{0}} \approx \frac{\Delta \mathrm{CBF}}{\mathrm{CBF}_{0}} .
$$

Since $\Delta v / v_{0}$ laser speckle imaging underestimates CBF by $<5 \%,{ }^{45}$ in this work both quantities were assumed to be equal. Flow images then underwent the same spatial filtering as the hemoglobin images, and images from all three different contrasts were further resized to one quarter of the original size due to memory constraints.

\subsection{Cerebral Metabolic Rate of Oxygen}

Changes in the cerebral metabolic rate of oxygen consumption $\left(\mathrm{CMRO}_{2}\right)$ were calculated from the images of changes in $\mathrm{CBF}$, total hemoglobin (HbT) and HbR using the steady-state relationship ${ }^{46,47}$ :

$$
\frac{\Delta \mathrm{CMRO}_{2}}{\Delta \mathrm{CMRO}_{2,0}}=\frac{\left(1+\frac{\Delta \mathrm{CBF}}{\mathrm{CBF}_{0}}\right)\left(1+\gamma_{R} \frac{\Delta \mathrm{HbR}}{\mathrm{HbR}_{0}}\right)}{\left(1+\gamma_{T} \frac{\Delta \mathrm{HbT}}{\mathrm{HbT}_{0}}\right)}-1 .
$$

A central hypothesis of this relation is the absence of transients with decoupled hemodynamic components. Here, since resting-state networks were identified from data filtered between 0.009 and $0.08 \mathrm{~Hz}$ (see below), we hypothesized that for these networks, this relationship was maintained. The constants $\gamma_{R}$ and $\gamma_{T}$ were both assumed to be 1 , which is within a physiologically plausible range $(0.75$ to 1.25$){ }^{46}$

\subsection{Functional Connectivity}

In this paper, we followed the procedure outlined by White et al. ${ }^{29}$ to produce seed-based correlation maps as a method for studying one aspect of functional connectivity; the steps are briefly described below.

Multimodal recording sessions (optical and electrical) of 15 min were carried out in resting-state conditions: Two sessions 
were performed before the injection of 4-AP and two more consecutive sessions were carried out $\sim 5 \mathrm{~min}$ after the release of the epileptogenic compound in the somatosensory cortex, giving a total of four sessions per mouse. Time courses of every pixel were temporally band-pass filtered (zero phase-shift fourth-order Butterworth filter) at 0.009 to $0.08 \mathrm{~Hz}$, according to previous functional connectivity studies. ${ }^{28,29}$ After temporal filtering, each pixel time trace was downsampled from 5 to $1 \mathrm{~Hz}$.

A global brain signal was created from the average of all the pixel time traces. In order to account for coherent variability common to all pixels, this global brain signal was regressed from each pixel time course, using a general linear model from the package statistical parametrical mapping ${ }^{48}$ (SPM8, www.fil .ion.ucl.ac.uk/spm) running on a Matlab (The MathWorks, Natick, MA) platform.

All seeds were manually placed $a$ priori using the coordinates corresponding to left and right frontal, cingulate, motor, somatosensory, cingulated, and visual cortices, ${ }^{29}$ as shown in Fig. 1(b). Seed time-courses were computed as the mean time course of the pixels within a 3.5-pixel $(0.2 \mathrm{~mm})$ radius from the seed locus. An example of seed time-trace during seizure is illustrated in Fig. 1(d). This seed is placed at the epileptic focus and its location is indicated with a dotted circle in Fig. 1(b). An increase in $\mathrm{HbO}_{2}$ and a correspondent decrease in $\mathrm{HbR}$ is observed at the seizure onset.

With the unilateral application of 4-AP, we expect bilateral differences between brain regions so the metric used to evaluate functional connectivity was a regional bilateral functional correlation, defined as the correlation between each seed time course and its contralateral homologue, yielding six values for each mouse. The Pearson's coefficient $r$-values were converted to Fisher $Z$ measures using $Z(r)-\frac{1}{2} \ln [(1+r) /(1-r)]$ before performing the random effect rank sum tests. Since the assumptions for normal distributions might not hold in this study, significance was determined by the Wilcoxon rank sum test. $P$ values were corrected for multiple comparisons using false discovery rate (FDR) adjustment. Adjusted values were considered significant at $p<0.05$.

\section{Results}

\subsection{Identification of Functional Networks}

Functional networks were identified prior to 4-AP injection and in agreement with previous literature ${ }^{29}$ across mice for $\mathrm{HbO}_{2}$ (see Fig. 2 for representative maps of four sample mice) albeit with a stronger lateralization of some networks.

With multispectral acquisition, the same procedure was also performed for $\mathrm{HbR}$ and $\mathrm{CBF}$ estimated from speckle imaging; no significant change in spatial network organization was observed (data not shown). In one experiment, we performed the network analysis using a higher frame rate and compared with a similar analysis performed by dropping camera frames to investigate the effect of acquisition frame rate. No significant difference was observed confirming that the chosen $5-\mathrm{Hz}$ acquisition rate was adequate (data not shown).

\subsection{Changes in Functional Connectivity After Injection of 4-AP}

Out of nine mice, five showed clear seizure-like activity (see Table 1), while four showed high-intensity spiking activity. Seizure-like activity was characterized by fast rhythmic spiking

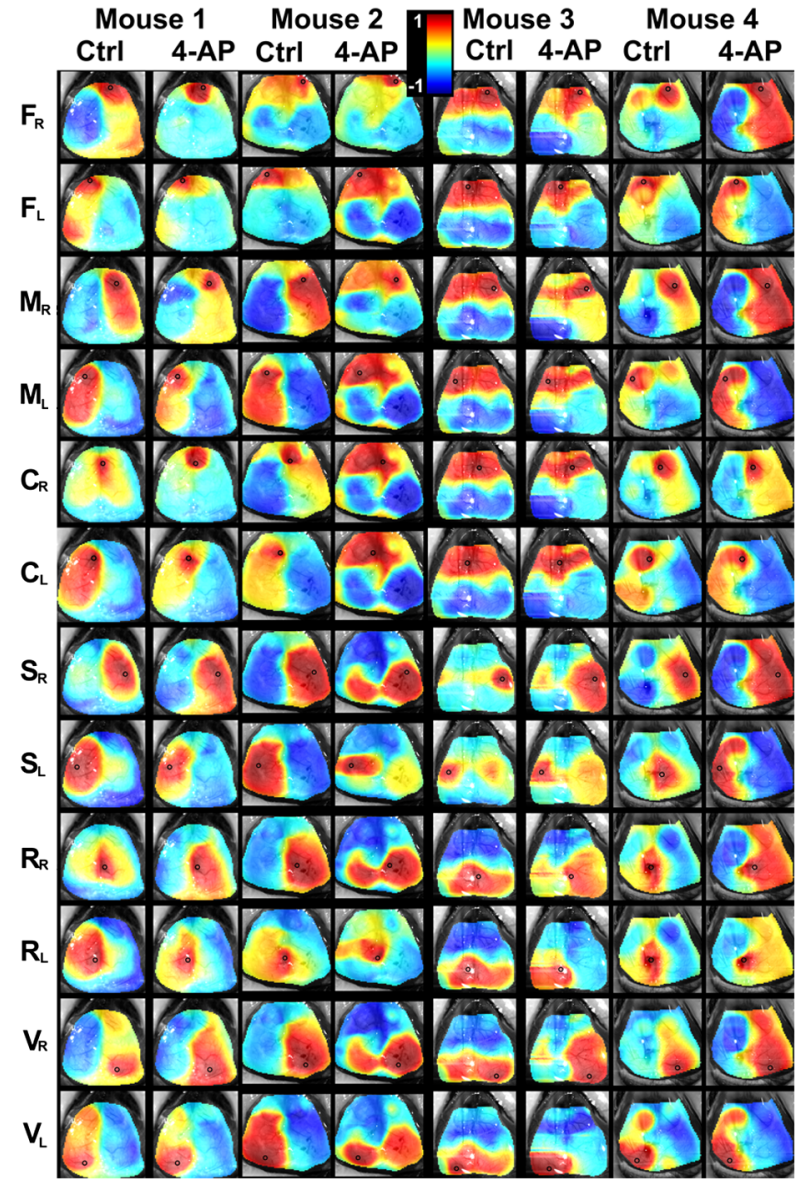

Fig. 2 Seed-based $\mathrm{HbO}_{2}$ correlation maps for four mice. One control session and one post-4-AP injection session are displayed for each mouse. (F: frontal cortex, M: motor cortex, C: cingulate cortex, S: somatosensory cortex R: retrosplenial cortex, V: visual cortex; subscripts L and $\mathrm{R}$ refer to left or right hemisphere, respectively.) The scale for all correlation maps is from $r=-1$ to 1 . Maps are shown overlaid on the anatomical image of the brain, acquired with green light. Seeds placement and sizes are indicated with black circles.

activity of increasing amplitude and decreasing frequency, evolving into rhythmic spike and slow wave activity prior to gradual offset [see an example in Fig. 1(c)], while spiking activity presents fewer than 10 spikes per burst (data not shown). All mice were combined in the analysis of this section. To assess the impact of acute seizures and epileptiform activity on restingstate networks, resting-state sessions acquired following the injection of 4-AP were analyzed following the same methodology as above.

The correlations between homologous seeds were investigated and compared before and after the injection of neurotoxin. Data extracted from the $\mathrm{HbO}_{2}$ time course showed a significant difference in retrosplenial seeds [Fig. 3(a)]. For HbR contrast, there was no significant difference in any paired seeds' time trace [Fig. 3(b)], although a decrease in functional connectivity was seen on the somatosensory region, a trend also seen in flow and $\mathrm{CMRO}_{2}$ data. Seeds of the cingulate, somatosensory, and retrosplenial cortex saw significant changes when analyzed with CBF contrast [Fig. 3(c)]: a decreased bilateral functional connectivity was observed in the somatosensory seeds while increased connectivity was seen in the cingulate and retrosplenial regions. 
Table 1 Seizure data. ${ }^{\mathrm{a}}$

\begin{tabular}{|c|c|c|c|c|c|}
\hline \multirow[b]{2}{*}{ Subject ID } & \multicolumn{2}{|c|}{ Seizure duration (s) } & \multicolumn{2}{|c|}{ Percentage duration in seizure state $(\%)$} & \multirow{2}{*}{$\frac{\text { Average seizure duration }(\mathrm{s})}{\text { Both sessions }}$} \\
\hline & Session 1 & Session 2 & Session 1 & Session 2 & \\
\hline 1 & $45,43,33,32$ & $35,32,27$ & $5.2,4.9,3.8,3.7$ & $4,3.7,3.1$ & 35.3 \\
\hline 3 & $27,33,34$ & - & $2.8,3.4,3.5$ & - & 31.3 \\
\hline 4 & 114 & 71,112 & 13.2 & $8.2,12.9$ & 99 \\
\hline 7 & $38,30,30$ & 33,32 & $4.4,3.4,3.4$ & $3.8,3.7$ & 32.6 \\
\hline 9 & 130 & 161 & 15 & 18.6 & 145.5 \\
\hline
\end{tabular}

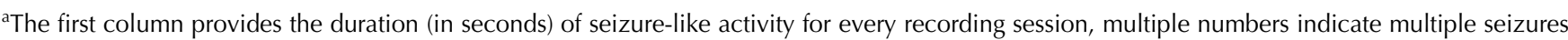
recorded; the second column shows the percentage of the recording session that belongs to seizure-like activity; finally the last column indicates the average duration of seizures per subject.

To assess whether these hemodynamic changes were associated with metabolic consumption patterns, we computed $\mathrm{CMRO}_{2}$ using steady-state formulas given the low-frequency fluctuations frequently documented in functional connectivity studies. ${ }^{10,49-51}$ The data shows no significant differences [Fig. 3(D)]. However there was a decrease in functional connectivity in the somatosensory and retrosplenial cortex, albeit not significant after FDR adjustment. All results were evaluated with a Wilcoxon rank sum test.

Functional networks were derived from low-frequency filtered imaging data. We were also interested to measure correlation between raw signals, before and after seizures, to observe the degree of disruption in the whole signal prior to regression by the common signal (data not shown). The data shows that all seeds are positively correlated, indicating the presence of a common signal to all the pixels' time course during epileptic events, hence the need to regress a global brain signal, obtained from the average time trace of all the pixels marked as belonging to the brain.

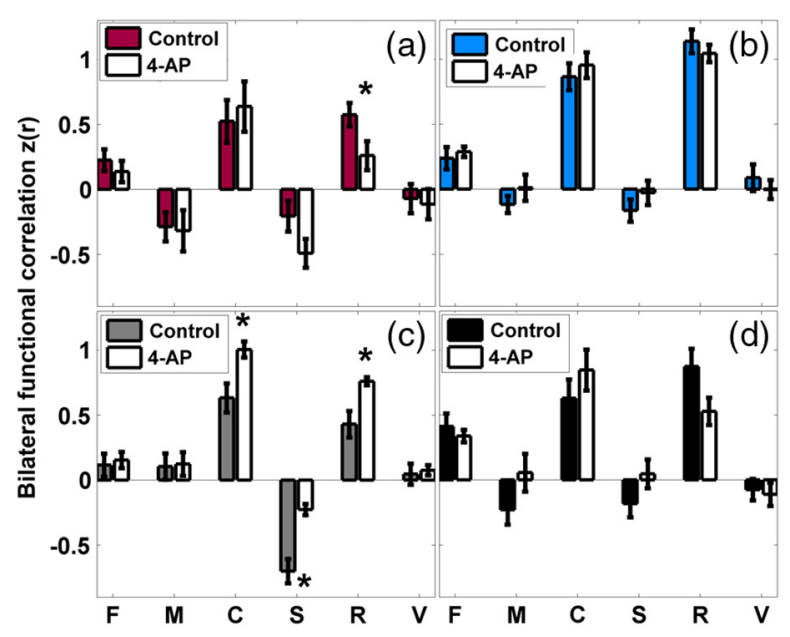

Fig. 3 Regional bilateral functional correlation before and after the 4-AP injection, analysis done for every seed time-trace and its contralateral part. Contrasts shown: (a) $\mathrm{HbO}_{2}$, (b) $\mathrm{HbR}$, (c) cerebral blood flow $(\mathrm{CBF})$, and (d) cerebral metabolic rate of oxygen consumption $\left(\mathrm{CMRO}_{2}\right) . * p<0.05$, FDR corrected. Standard error bars shown $(\sigma / \sqrt{ } N)$, with $N=9$.

\subsection{Correlation Between Seizure Duration and Functional Connectivity}

Restricting the analysis to the mice showing clear seizure activity, we aimed to investigate whether seizure duration impacted network changes. To calculate the changes of bilateral correlation, we subtracted the bilateral correlation during control sessions, $z_{0}(r)$, from the bilateral correlation during the post 4-AP injection sessions, $z_{4 \mathrm{AP}}(r)$, and plotted this change versus the average seizure duration of the 4-AP sessions, as illustrated in Fig. 4. Overall correlations between seizure duration and changes in bilateral correlations were moderate. For the somatosensory cortex, where the toxin was injected, there was a positive correlation between the duration of the seizure and the changes in bilateral correlation. This same positive correlation was observed from the data extracted from the frontal cortex seeds. For other cortical regions, i.e., motor, cingulate, retrosplenial, and visual, a reverse effect was observed: the changes were negatively correlated with the seizure duration. For CBF contrast, the correlation showed to be significant in the somatosensory $\left(r^{2}=0.52\right)$ and retrosplenial $\left(r^{2}=0.75\right)$ regions. HbR contrast had a significant correlation for retrosplenial seeds $\left(r^{2}=0.50\right)$, while $\mathrm{HbO}_{2}$ displayed a moderate (not significant) correlation for the motor seeds $\left(r^{2}=0.41\right)$. The average length of seizures per subject was $68.7 \pm 51 \mathrm{~s}$. All recording sessions were $863 \mathrm{~s}$ long. Table 1 provides the duration of seizure-like activity for every recording session, the percentage of the recording session that belongs to seizure-like activity, and the average duration of seizures per subject.

\section{Discussion}

In this work, we investigated regional bilateral functional connectivity using a seed-based correlation analysis; functional connectivity was evaluated before and after the injection of epileptogenic 4-AP in the somatosensory cortex.

Using $\mathrm{HbO}_{2}$ contrast, significant differences in functional connectivity were found in the retrosplenial cortex. With HbR contrast, no seeds showed a statistically significant difference though most correlations decreased. In the case of relative blood flow contrast, a significant difference was observed in the cingulate, somatosensory, and retrosplenial regions. The only paired seeds to show a significant decrease of functional connectivity were in the somatosensory cortex, while the retrosplenial and cingulate exhibited an increase in functional 


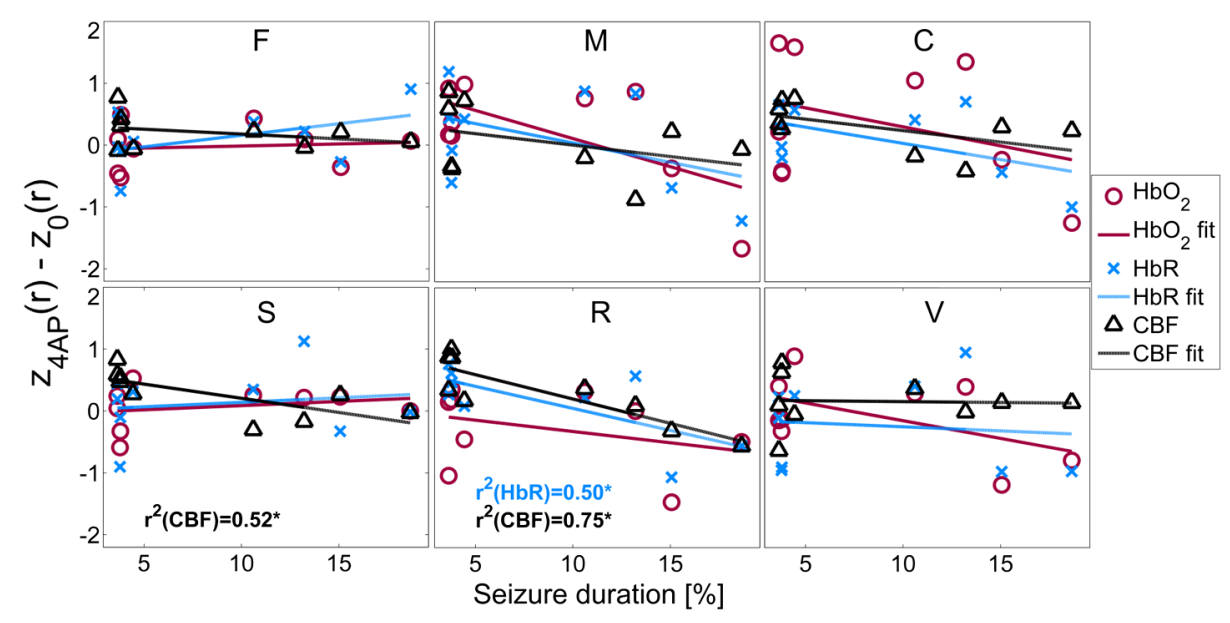

Fig. 4 Changes in bilateral functional correlation plotted versus seizure duration (expressed as a percentage of the recording session) for different cortical regions and different contrasts.

correlation; we hypothesize this increase may be explained by the proximity of these last two regions to the superior sagittal sinus, so that their behavior is dominated by increased venous drainage at epileptic activity. For the estimated $\mathrm{CMRO}_{2}$, no significant changes were found in functional connectivity measures.

Due to the proximity of cingulate and retrosplenial seeds to the site of 4-AP injection, the significant changes in the bilateral functional connectivity of these regions could be attributed to the proximity to the injection site, suggesting that the neurotoxin 4-AP might have propagated to these regions. However, at the site of injection, homologous connectivity decreased, suggesting a decoupling of hemispheric connections linking the somatosensory areas while at the cingulate and retrosplenial sites, connectivity increased. An explanation might be a potential compensatory role for nearby regions (increasing their connectivity) to compensate for a reduction in the somatosensory homologous synchronization. A similar phenomenon was observed in Ref. 52, where following stroke in the sensory cortex in mice, a functional reorganization in nearby areas was observed but after much longer periods ( 8 weeks). Another hypothesis is that hemodynamic changes observed here may reflect a local redistribution of blood flow associated with the high metabolic activity at the seizure location.

\subsection{Acute Seizure Activity Leads to Both Decrease and Increase in Functional Connectivity}

In the case of $\mathrm{HbO}_{2}$, a significant decrease of functional connectivity was observed in retrosplenial cortex. However, the difference between control and post-4-AP injection were not significant in HbR networks. In blood flow contrast, a significant increase of functional connectivity was observed in both cingulate and retrosplenial paired seeds, and there was a significant decrease for somatosensory cortex.

Previous literature using fMRI ${ }^{11,20,21,24}$ mostly documented reduced functional connectivity in several functional networks in epileptic patients measured at rest. While a clear distinction is required with current experiments which focused on restingstate networks during acute epileptiform events, the picture emerging from this work indicates a complex interplay between the different components of the hemodynamic response leading to both increases and decreases in connectivity in our mouse model. While HbR, which closely correlates to BOLD-fMRI, saw a nonsignificant decrease in connectivity in most seeds (4/5), $\mathrm{HbO}_{2}$ and $\mathrm{CBF}$ saw their bilateral correlations move in opposite directions in seeds closest to the injection site (somatosensory and retrosplenial, for which changes were significant). Hemodynamic component dependent correlation changes indicate a potential uncoupling of flow and metabolism, even in low-frequency networks.

These observations suggest that using a single modality, e.g., fMRI BOLD, to investigate resting-state network in epilepsy may remain limited without controlling for flow or oxygenation. Whether the observed changes are due to changes in physiology (however, no significant change was observed on our ECG), high metabolic demand at the seizure location, or neural activity remains to be investigated. Further work with a chronic model investigating networks outside of epileptic periods may help reveal more coherent changes across hemodynamic components.

\subsection{Correlation Between Electrophysiological Activity and Resting-State Correlations}

Since electrophysiology was measured simultaneously, we could investigate the effect of seizure duration on pairwise network correlations. Our results showed a dependence of the changes in bilateral functional correlations on seizure duration but remained mitigated due the low values of $r^{2}$ obtained in this analysis. Whether these changes are also present in subacute conditions, during simple spiking events, and the cause for network changes observed in patients remain to be studied. Our results are further limited by the fact that electrophysiology was measured in the somatosensory cortex; it may be that similar activity measured at other seeds may lead to a different outcome. Keeping the skull intact apart from the injection site allowed studying an almost intact cortex but the absence of multiple LFP recording sites implied that the spatial dependence of the LFP response remained a potentially confounding variable that was not investigated.

\subsection{Metabolic Consumption Reflects Hemodynamic Changes}

$\mathrm{CMRO}_{2}$ is potentially the closest marker to neuronal activity. Here, we hypothesized that the steady-state relation between hemodynamic signals to estimate $\mathrm{CMRO}_{2}$ held for the 
resting-state networks due to their low-frequency support. Assuming this hypothesis to be valid and comparing network correlation changes obtained by $\mathrm{CMRO}_{2}$, our analysis displayed changes that followed similar trends as changes in HbR, except for the visual and frontal seeds where small differences were observed. This observation may in turn support HbR-weighted fMRI-BOLD as a proxy for resting-state network in epilepsy despite the known physiological confounds and hemodynamic network uncoupling displayed here.

\section{Conclusion}

Our original aim was to investigate the potential of OIS to study epileptic networks. Combining OIS with laser speckle imaging allows for simultaneous imaging of changes in $\mathrm{HbO}_{2}, \mathrm{HbR}$, $\mathrm{CBF}$, and potentially $\mathrm{CMRO}_{2}$, providing a good set of hemodynamic data for functional connectivity studies during acute seizures. In our work, following 4-AP injection in the somatosensory cortex of mice, we observed significant changes in networks derived from spatial seeds near the injection site. Changes were not uniform across hemodynamic components, signaling a complex picture with a potential decoupling of flow and metabolism within the low-frequency networks. This study thus supports the need for a more extensive investigation and characterization of epileptic networks encompassing more than one hemodynamic measurement.

There are several limitations to be mentioned; first and foremost our results were obtained during acute epileptiform events in a relatively small sample size. They cannot therefore be directly compared with connectivity studies in humans typically obtained during rest periods devoid of acute epileptic events. Thus care must be taken when interpreting the current results since several physiological factors may be confounding the observed neuronal activity. Further work will aim to investigate larger populations of chronic animal models of epilepsy in conditions that are closer to ongoing human resting-state studies.

\section{Acknowledgments}

This research was supported by NSERC Discovery grant to F. Lesage. E. Guevara acknowledges financial support from Mexican National Science and Technology Council (CONACYT) through scholarship no. 304501. The authors wish to thank Tri Truong Van for his assistance in animal preparation and Mingrui Zhao for advice with 4-AP injections.

\section{References}

1. E. R. Kandel, J. H. Schwartz, and T. M. Jessell, Principles of Neural Science, 3rd ed., Elsevier, New York (1991).

2. World Health Organization, Neurological Disorders: Public Health Challenges, WHO Press, Geneva, Switzerland (2006).

3. F. A. Gibbs, E. L. Gibbs, and W. G. Lennox, "Epilepsy: a paroxysmal cerebral dysrhythmia," Brain 60(4), 377-388 (1937).

4. J. Gotman et al., "Generalized epileptic discharges show thalamocortical activation and suspension of the default state of the brain," Proc. Natl. Acad. Sci. U.S.A. 102(42), 15236-15240 (2005).

5. A. Gallagher et al., "Non-invasive pre-surgical investigation of a 10 year-old epileptic boy using simultaneous EEG-NIRS," Seizure 17(6), 576-582 (2008).

6. D. K. Nguyen et al., "Non-invasive continuous EEG-fNIRS recording of temporal lobe seizures," Epilepsy Res. 99(1-2), 112-126 (2012).

7. M. Zhao et al., "Focal increases in perfusion and decreases in hemoglobin oxygenation precede seizure onset in spontaneous human epilepsy," Epilepsia 48(11), 2059-2067 (2007).
8. F. Morrell and L. deToledo-Morrell, "From mirror focus to secondary epileptogenesis in man: an historical review," Adv. Neurol. 81, 11-23 (1999).

9. C. Bruehl, G. Hagemann, and O. W. Witte, "Uncoupling of blood flow and metabolism in focal epilepsy," Epilepsia 39(12), 1235-1242 (1998).

10. M. D. Fox and M. E. Raichle, "Spontaneous fluctuations in brain activity observed with functional magnetic resonance imaging," Nat. Rev. Neurosci. 8(9), 700-711 (2007).

11. A. B. Waites et al., "Functional connectivity networks are disrupted in left temporal lobe epilepsy," Ann. Neurol. 59(2), 335-343 (2006).

12. F. Wendling et al., "Interictal to ictal transition in human temporal lobe epilepsy: insights from a computational model of intracerebral EEG," J. Clin. Neurophysiol. 22(5), 343-356 (2005).

13. F. Mormann et al., "Epileptic seizures are preceded by a decrease in synchronization," Epilepsy Res. 53(3), 173-185 (2003).

14. A. Aarabi, F. Wallois, and R. Grebe, "Does spatiotemporal synchronization of EEG change prior to absence seizures?," Brain Res. 1188, 207-221 (2008).

15. S. C. Ponten, F. Bartolomei, and C. J. Stam, "Small-world networks and epilepsy: graph theoretical analysis of intracerebrally recorded mesial temporal lobe seizures," Clin. Neurophysiol. 118(4), 918-927 (2007).

16. K. Schindler et al., "Assessing seizure dynamics by analysing the correlation structure of multichannel intracranial EEG," Brain 130(Pt 1), 65-77 (2007).

17. G. Bettus et al., "Enhanced EEG functional connectivity in mesial temporal lobe epilepsy," Epilepsy Res. 81(1), 58-68 (2008).

18. M. Righi et al., "Detection of signs of brain dysfunction in epileptic children by recognition of transient changes in the correlation of seizure-free EEG," Brain Topogr. 21(1), 43-51 (2008).

19. L. Douw et al., "'Functional connectivity' is a sensitive predictor of epilepsy diagnosis after the first seizure," PLoS One 5(5), e10839 (2010).

20. C. Luo et al., "Disrupted functional brain connectivity in partial epilepsy: a resting-state fMRI study," PLoS One 7(1), e28196 (2012).

21. K. Mankinen et al., "Connectivity disruptions in resting-state functional brain networks in children with temporal lobe epilepsy," Epilepsy Res. 100(1-2), 168-178 (2012).

22. Z. Wang et al., "Altered resting state networks in epileptic patients with generalized tonic-clonic seizures," Brain Res. 1374, 134-141 (2011).

23. L. Frings et al., "Remote effects of hippocampal damage on default network connectivity in the human brain," J. Neurol. 256(12), 2021-2029 (2009).

24. W. Liao et al., "Default mode network abnormalities in mesial temporal lobe epilepsy: a study combining fMRI and DTI," Hum. Brain Mapp. 32(6), 883-895 (2011).

25. Z. Zhang et al., "Impaired attention network in temporal lobe epilepsy: a resting FMRI study," Neurosci. Lett. 458(3), 97-101 (2009).

26. N. Voges et al., "Modeling of the neurovascular coupling in epileptic discharges," Brain Topogr. 25(2), 136-156 (2012).

27. P. Pouliot et al., "Nonlinear hemodynamic responses in human epilepsy: a multimodal analysis with fNIRS-EEG and fMRI-EEG," J. Neurosci. Methods 204(2), 326-340 (2012).

28. A. W. Bero et al., "Bidirectional relationship between functional connectivity and amyloid- $\beta$ deposition in mouse brain," J. Neurosci. 32(13), 4334-4340 (2012).

29. B. R. White et al., "Imaging of functional connectivity in the mouse brain," PLoS One 6(1), e16322 (2011).

30. P. F. Fabene et al., "Structural and functional MRI following 4-aminopyridine-induced seizures: a comparative imaging and anatomical study," Neurobiol. Dis. 21(1), 80-89 (2006).

31. M. Zhao et al., "Spatiotemporal dynamics of perfusion and oximetry during ictal discharges in the rat neocortex," J. Neurosci. 29(9), 2814-2823 (2009).

32. M. Zhao et al., "Preictal and ictal neurovascular and metabolic coupling surrounding a seizure focus," J. Neurosci. 31(37), 13292-13300 (2011).

33. G. Bettus et al., "Interictal functional connectivity of human epileptic networks assessed by intracerebral EEG and BOLD signal fluctuations," PLoS One 6(5), e20071 (2011).

34. O. Moldestad et al., "Tracheotomy improves experiment success rate in mice during urethane anesthesia and stereotaxic surgery," J. Neurosci. Methods 176(2), 57-62 (2009). 
35. S. Dubeau et al., "Cerebrovascular hemodynamic correlates of aging in the Lou/c rat: A model of healthy aging," Neurolmage 56(4), 1892-1901 (2011).

36. A. K. Dunn et al., "Simultaneous imaging of total cerebral hemoglobin concentration, oxygenation, and blood flow during functional activation," Opt. Lett. 28(1), 28-30 (2003).

37. D. T. Delpy et al., "Estimation of optical pathlength through tissue from direct time of flight measurement," Phys. Med. Biol. 33(12), 1433-1442 (1988).

38. A. K. Dunn et al., "Spatial extent of oxygen metabolism and hemodynamic changes during functional activation of the rat somatosensory cortex," Neuroimage 27(2), 279-290 (2005).

39. M. Kohl et al., "Physical model for the spectroscopic analysis of cortical intrinsic optical signals," Phys. Med. Biol. 45(12), 3749-3764 (2000).

40. S. Prahl, "Optical absorption of hemoglobin" (1999), <http://omlc.ogi .edu/spectra/hemoglobin/> (8 October 2009).

41. N. Brieu et al., "Characterization of the hemodynamic response in the rat lumbar spinal cord using intrinsic optical imaging and laser speckle," J. Neurosci. Methods 191(2), 151-157 (2010).

42. D. A. Boas and A. K. Dunn, "Laser speckle contrast imaging in biomedical optics," J. Biomed. Opt. 15(1), 011109 (2010).

43. A. K. Dunn et al., "Dynamic imaging of cerebral blood flow using laser speckle," J. Cereb. Blood Flow Metab. 21(3), 195-201 (2001).

44. J. D. Briers, "Laser Doppler, speckle and related techniques for blood perfusion mapping and imaging," Physiol. Meas. 22(4), R35-R66 (2001).
45. J. Luckl et al., "The biological effect of contralateral forepaw stimulation in rat focal cerebral ischemia: a multispectral optical imaging study," Front Neuroenerget. 2(pii), 19 (2010).

46. M. Jones et al., "Concurrent optical imaging spectroscopy and laserDoppler flowmetry: the relationship between blood flow, oxygenation, and volume in rodent barrel cortex," Neuroimage 13(6 Pt 1), 1002-1015 (2001).

47. J. Mayhew et al., "Spectroscopic analysis of neural activity in brain: increased oxygen consumption following activation of barrel cortex," Neurolmage 12(6), 664-675 (2000).

48. K. J. Friston et al., Statistical Parametric Mapping: The Analysis of Functional Brain Images, 1st Ed., Academic Press, Great Britain (2006).

49. B. Biswal et al., "Functional connectivity in the motor cortex of resting human brain using echo-planar MRI," Magn. Reson. Med. 34(4), 537-541 (1995).

50. B. B. Biswal et al., "Toward discovery science of human brain function," Proc. Natl. Acad. Sci. 107(10), 4734-4739 (2010).

51. M. D. Greicius et al., "Functional connectivity in the resting brain: a network analysis of the default mode hypothesis," Proc. Natl. Acad. Sci. U.S.A. 100(1), 253-258 (2003).

52. C. E. Brown et al., "In Vivo voltage-sensitive dye imaging in adult mice reveals that somatosensory maps lost to stroke are replaced over weeks by new structural and functional circuits with prolonged modes of activation within both the peri-infarct zone and distant sites," J. Neurosci. 29(6), 1719-1734 (2009). 